\title{
Prophylactic or therapeutic doses of heparins for COVID-19 infection? A retrospective study
}

\author{
Francesco Bolzetta ${ }^{1} \cdot$ Monica Maselli ${ }^{1} \cdot$ Marino Formilan ${ }^{1} \cdot$ Flavio Busonera $^{1} \cdot$ Paolo Albanese $^{1}$. \\ Federica Chiaromanni ${ }^{1}$. Antonietta Romano ${ }^{1} \cdot$ Nicola Veronese $^{2}$ (I)
}

Received: 8 August 2020 / Accepted: 30 October 2020 / Published online: 16 November 2020

(c) Springer Nature Switzerland AG 2020

\begin{abstract}
Background Coronavirus disease 19 (COVID-19) is a global outbreak. COVID-19 patients seem to have relevant coagulative abnormalities, even if they are not typical of disseminated intravascular coagulopathy (DIC) of the kind seen in septicaemia. Therefore, anticoagulant therapy with heparins is increasing in interest for a clinical approach to these patients, particularly if older. Studies comparing if prophylactic doses are more effective than therapeutic ones are still missing.

Methods Data were collected in the Geriatric Section of the Dolo Hospital, ULSS 3 "Serenissima", Venice from 31st March to 01st May 2020. Heparins (calciparin, fondaparinux, enoxaparine) were divided into prophylactic or therapeutic doses. People previously treated with oral anticoagulants were removed. Vital status was assessed using administrative data. Cox's regression analysis, adjusted for potential confounders, was used for assessing the strength of the association between heparins and mortality. The data were reported as hazard ratio (HR) with $95 \%$ confidence intervals (CIs).

Results 81 older people (mean age 84.1 years; females $=61.9 \%$ ) were included. No significant differences in terms of demographic and clinical characteristics emerged between people treated with prophylactic or therapeutic doses, including age, gender, X-rays findings or severity of disease. Therapeutic doses were not associated to a better survival rate (HR 1.06; 95\% CI $0.47-2.60 ; p=0.89$ ), even after adjusting for 15 confounders related to mortality (HR $0.89 ; 95 \%$ CI $0.30-2.71 ; p=0.84$ ). Conclusions Our paper indicates that in older people affected by COVID-19 there is no justification for using therapeutic doses instead of prophylactic ones, having a similar impact on mortality risk.
\end{abstract}

Keywords COVID-19 $\cdot$ Heparin $\cdot$ Mortality

\section{Introduction}

Coronavirus disease 19 (COVID-19) is a global outbreak. COVID-19 patients seem to have relevant coagulative abnormalities [1]. These alterations could appear both as thrombotic microangiopathy or disseminated intravascular coagulation, like in other severe infections [1]. Moreover, many patients also develop venous or arterial thrombosis [1].

Nicola Veronese

ilmannato@gmail.com; nicola.veronese1@aulss3.veneto.it

1 Medical Department, Geriatric Unit, Azienda ULSS [Unità Locale Socio Sanitaria] 3 "Serenissima”, Dolo-Mirano District, Venice, Italy

2 Primary Care Department, Azienda ULSS 3 [Unità Locale Socio Sanitaria] "Serenissima”, Dolo-Mirano District, Via Murano, 17 Spinea, Venice, Italy
Patients who died for COVID-19 pneumonia showed significantly increased higher levels of D-dimer and fibrin degradation products (FDP), longer prothrombin time (PT), with also later lower value of fibrinogen and anti-thrombin. All these data revealed an increased coagulation activation in COVID-19 patients, with an increased risk of death [2]. Moreover, severe patients are also often immobilized, with an elevated additional risk for venous thromboembolism development. Therefore, anticoagulant therapy with heparins is increasing in interest for a clinical approach to these patients, particularly if older. Anticoagulant therapy seems to be important to reduce fibrin deposition, microthrombi formation and prothrombotic state in these patients [2].

In a recent paper, the administration of Low Molecular Weight Heparin (LMWH) at prophylactic dose was highly recommended for all patients with COVID-19 infection that required hospitalization, in absence of contraindications [3]. Nevertheless, is not clear if the benefit in term of survivance 
with prophylactic doses of LMWH is limited only to patients that develop sepsis-induced coagulopathy during the course of COVID-19 infection [4]. It is also possible that patients with more severe infection could benefit from even higher doses of LMWH, several trials are investigating that point [1].

Studies comparing if prophylactic doses are more effective than therapeutic ones are still missing. Given this background, the aim of the present paper is to investigate the benefit in terms of mortality between the prophylactic or therapeutic dose of LMWH in older patients and COVID-19.

\section{Methods}

\section{Participants}

Data were collected in the Geriatric Section of the Dolo Hospital, ULSS 3 "Serenissima", Venice from 31st March to 01st May 2020. The protocol of the study was approved by our Local Ethical Committee.

\section{COVID-19 diagnosis}

To all the patients, a nasopharyngeal swab test with an RTPCR assays (Copan UTM System, Copan, Italy) for the identification of SARS-CoV-2 was administered.

\section{Exposure: heparins}

People previously treated with oral anticoagulants (e.g. for atrial fibrillation) were removed. Heparins (calciparin, fondaparinux, enoxaparin) were then divided in prophylactic or therapeutic doses. The decision for treating a patient with prophylactic or therapeutic doses was based on clinical, bio-humoral, radiological findings, giving higher doses of heparins to people at higher risk of mortality [5], since no univocal guidelines exist for therapy with heparins for COVID-19 [5]. The mean duration of treatment was 11 days.

\section{Outcome: mortality}

Vital status was assessed using administrative data, recording the date of death. The mean follow-up period was 31 days.

\section{Clinical parameters}

For the aims of this study, we collected several information, including (1) Demographics (age, sex); (2) clinical signs at the admission (temperature, systolic and diastolic blood pressure, Arterial Oxygen saturation, $\mathrm{SpO}_{2}$ ); (3) presence of comorbidities, verified through medical history, medications, laboratory and radiological tests; (4) X-ray findings suggestive of COVID-19 infection and divided in pulmonary thickening and/or interstitial pneumonia; (5) bio-humoral findings: we collected several bio-humoral laboratory tests: for the aims of this paper, we have reported only those more strongly related to heparin use. These measurements were made using standard laboratory procedures.

\section{Statistical analysis}

After verifying the normality of the continuous variables included, we reported the data as mean with standard deviations (SDs) or, in case of categorical variables, as percentages (\%). Independent groups $T$-test was used for continuous variables, Chi-square test (with Fisher's correction) for categorical parameters. For D-dimer, due to non-normal distribution, data are reported as median (with interquartile range) and the Kruskal-Wallis test was used for comparing any difference in the median.

For assessing the association between heparins' dosages and mortality, we used a Cox's regression analysis, adjusted for potential confounders. Confounders (i.e. age, sex, and presence of obesity, diabetes, cancer, COPD, dementia, Parkison's disease, renal failure, acute myocardial infarction, stroke, heart failure, high blood pressure, pressure sores, hepatic cirrhosis) were included in this model only if they reached, at the univariate analyses, a $p$-value $<0.10$. The data were reported as hazard ratio (HR) with their $95 \%$ confidence intervals (CIs).

Threshold of statistical significance was set to 5\%. All statistical analyses were performed with SPSS 21.0.

\section{Results}

Overall, 88 older people were recovered in our ward. After excluding 7 persons treated with oral anticoagulants, 81 older people (mean age 84.1 years; females $=61.9 \%$ ) were finally included.

Table 1 shows the most important descriptive findings, by a dose of LMWH used during the hospitalization. No significant differences in terms of demographic and clinical characteristics emerged between people treated with prophylactic or therapeutic doses, including age, gender, X-rays findings or presence of comorbidities. The only bio-humoral parameter significantly different was serum creatinine levels $(1.61 \pm 1.11$ in prophylactic doses vs. $1.22 \pm 0.52$ in therapeutic doses; $p$ value $=0.04$ ) (Table 1). No significant difference in the mean duration of the therapy emerged between the two groups ( $10.8 \pm 8.7$ vs. $10.7 \pm 8.3$ days; $p=0.94)$.

As shown in Fig. 1, the cumulative incidence of deaths was similar between subjects taking a prophylactic or therapeutic dose of LMWH, being 50\% in both groups (log rank 
Table 1 Descriptive findings of the participants included, by dose of LMWH

\begin{tabular}{|c|c|c|c|}
\hline Parameter & Prophylactic doses $(n=57)$ & $\begin{array}{l}\text { Therapeutically doses } \\
(n=24)\end{array}$ & $p$ value \\
\hline Age (years) & $84.1(11.9)$ & $87.1(8.0)$ & 0.25 \\
\hline Females $(\%)$ & 43.9 & 29.2 & 0.32 \\
\hline Temperature & $37.4(1.5)$ & $36.9(0.9)$ & 0.61 \\
\hline Systolic blood pressure & $125(29)$ & $126(25)$ & 0.99 \\
\hline Diastolic blood pressure & $76(16)$ & $76(13)$ & 0.99 \\
\hline $\mathrm{SpO}_{2}$ & $93.0(5.1)$ & $92.1(6.4)$ & 0.59 \\
\hline \multicolumn{4}{|c|}{ Comorbidities, in alphabetical order } \\
\hline Cancer (\%) & 7.0 & 12.5 & 0.42 \\
\hline Cirrhosis (\%) & 1.8 & 0.0 & 0.99 \\
\hline COPD $(\%)$ & 17.5 & 8.3 & 0.49 \\
\hline Dementia (\%) & 64.9 & 54.2 & 0.45 \\
\hline Diabetes $(\%)$ & 22.8 & 29.2 & 0.58 \\
\hline Heart failure (\%) & 3.5 & 4.2 & 0.99 \\
\hline Hypertension (\%) & 61.4 & 66.7 & 0.80 \\
\hline Obesity (\%) & 10.5 & 4.2 & 0.67 \\
\hline Parkinson's disease (\%) & 5.3 & 8.3 & 0.63 \\
\hline Pressure sores $(\%)$ & 15.8 & 4.2 & 0.27 \\
\hline Previous AMI (\%) & 7.0 & 8.3 & 0.99 \\
\hline Renal failure (\%) & 17.5 & 12.5 & 0.75 \\
\hline Stroke $(\%)$ & 7.0 & 4.2 & 0.99 \\
\hline X-ray findings & & & 0.15 \\
\hline Pulmonary thickening (\%) & 27.8 & 16.7 & \\
\hline Interstitial pneumonia (\%) & 33.3 & 45.8 & \\
\hline Both $(\%)$ & 22.2 & 29.2 & \\
\hline \multicolumn{4}{|l|}{ Main bio-humoral findings } \\
\hline D-dimer & $1651(640-2981)$ & $744(695-7489)$ & 0.29 \\
\hline Creatinine & $1.61(1.11)$ & $1.22(0.52)$ & 0.04 \\
\hline Hemoglobin & $12.2(2.4)$ & $12.7(2.1)$ & 0.43 \\
\hline Platelets & $215(84)$ & $245(91)$ & 0.15 \\
\hline
\end{tabular}

Data are reported as mean (with standard deviation) for continuous variables and percentages for categorical variables, except for D-dimer for which the data are reported as median (with interquartile range). Independent groups $T$-test was used for continuous variables (Kruskall-Wallis test for D-dimer), chi-square test (with Fisher's correction) for categorical parameters test, $p$ value $=0.89)$. Therapeutic doses were not associated to a better survival rate (HR 1.06; 95\% CI 0.47-2.60; $p=0.89$ ), even after adjusting for 15 confounders related to mortality (HR $0.89 ; 95 \%$ CI $0.30-2.71 ; p=0.84$ ).

\section{Discussion}

In the present retrospective study, including a group of older people affected by COVID-19, we reported no difference in terms of mortality between subjects taking a prophylactic or therapeutic dose of LMWH. This finding remained unaltered after adjustment for several potential confounders.

In a Chinese study involving 449 subjects with COVID19 a reduction in mortality in people treated with low doses of LMWH was observed only for patients with higher D-dimer level or sepsis-induced coagulopathy [6]. While the use of a prophylactic dose of LMWH in all COVID-19 patients is currently encouraged by several scientific associations [7], the use of higher doses is still debated.

The use of a therapeutic dose of LMWH could be suggested by the high incidence of venous thromboembolism in severe COVID-19 patients reported in the literature. In a recent paper, an incidence of $47 \%$ of venous thromboembolism was observed in a patient requiring intensive care unit for COVID-19, while in patients admitted to regular ward the incidence of symptomatic venous thromboembolism was $3 \%$, despite the use of a prophylactic dose of LMWH [8]. Similarly, in an Italian study the incidence of venous thromboembolism was $6.6 \%$ in patients hospitalized in the normal ward and $27.6 \%$ in intensive care unit [9]. 
Fig. 1 Survival curves by dose of LMWH

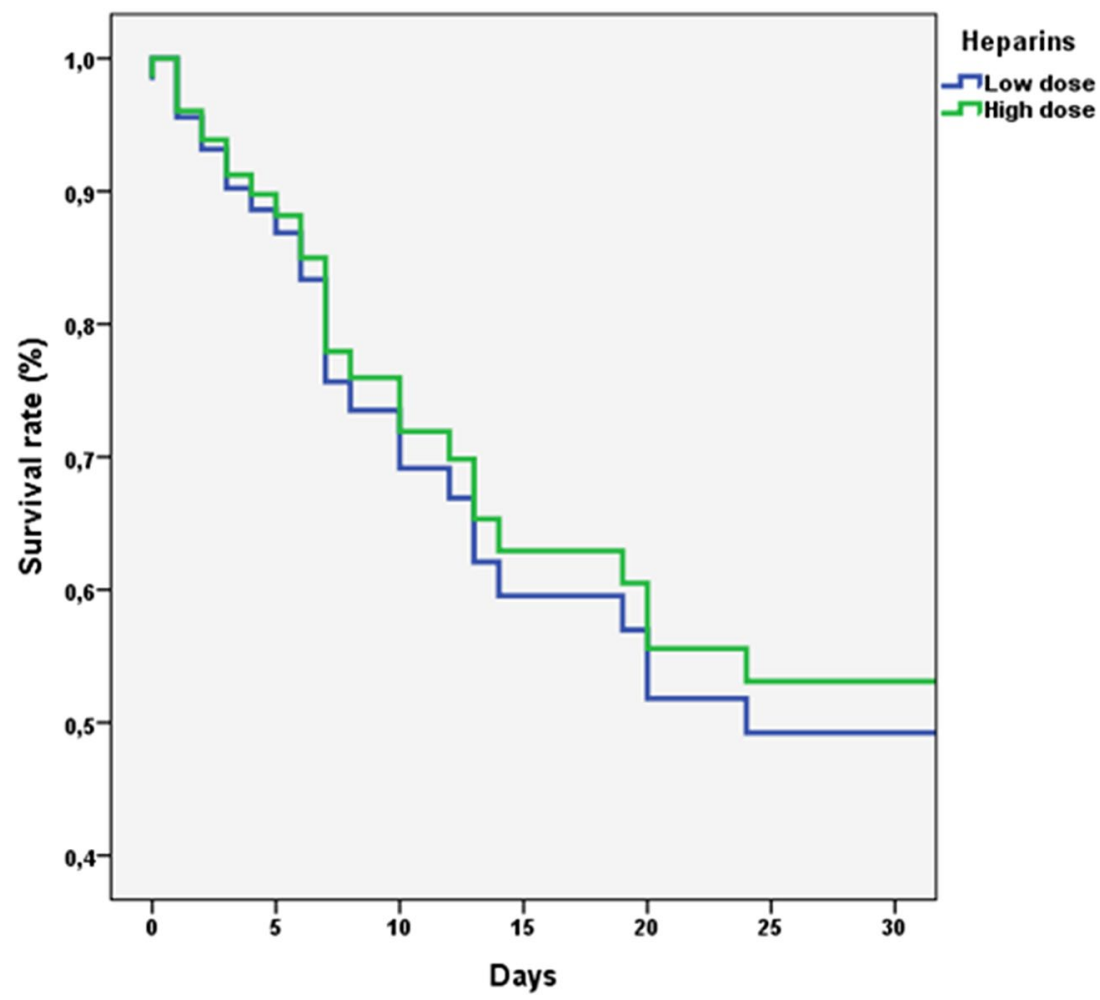

All these data, including our report, seem to highlight that maybe the protective effect of the therapeutic dose of LMWH in COVID-19 could be relevant only in selected or severe patients. Lacking definitive data on LMWH dose for COVID-19 patients, currently, there are only treatment suggestions elaborated by experts in the field of coagulation and based on a patient-tailored approach taking into account laboratory values, particularly D-dimer levels, comorbidities, risk factors, and severity of the infection [10-12].

The findings of our paper should be interpreted within its limitations. First, the retrospective nature of this study that can contain some biases. Second, we did not collect, during the follow-up, hemorrhagic events. Third, we did not calculate any prognostic or severity index that can be important in better tailoring therapy in older people. Third, to know some of the predictors of prognosis in such a frail population would be of importance, since some of these factors could be responsible at the same time of the dose prescription and of mortality. Finally, the sample size is limited (less than 100 participants) and with a short follow-up period. Despite these limitations, we believe that our paper has some strengths, in particular the fact that is the first study exploring this important clinical topic.

In conclusion, our study indicates that in older people affected by COVID-19 admitted to a regular ward, there is no justification for using therapeutic doses instead of prophylactic ones, having a similar impact on mortality risk. Future RCTs are needed to confirm our findings.
Author contributions Data analysis and statistical analysis: NV; data collection: MM, FB; drafting: FB, MF, FB; critical revision: FC, PA, AR.

Funding None.

Data availability Available upon request to the corresponding author.

Code availability All statistical analyses were performed with SPSS 21.0 .

\section{Compliance with ethical standards}

Conflict of interest None to declare.

Ethics approval In agreement with the current Italian law [Gazzetta Ufficiale della Repubblica Italiana, Serie Generale n. 76 del 31-032008], we informed our local Ethical Committee of this nature that is observational and collect the data regarding normal critical practice.

Consent to participate Informed consent was collected orally for hygienic reasons.

\section{References}

1. Levi M, Thachil J, Iba T et al (2020) Coagulation abnormalities and thrombosis in patients with COVID-19. Lancet Haematol 7:e438 
2. Arachchillage DR, Laffan M (2020) Abnormal coagulation parameters are associated with poor prognosis in patients with novel coronavirus pneumonia. J Thromb Haemost 18:1233-1234

3. Thachil J, Tang N, Gando S et al (2020) ISTH interim guidance on recognition and management of coagulopathy in COVID-19. J Thromb Haemost 18:1023-1026

4. Iba T, Di Nisio M, Levy JH et al (2017) New criteria for sepsisinduced coagulopathy [SIC] following the revised sepsis definition: a retrospective analysis of a nationwide survey. BMJ Open 7:e017046. https://doi.org/10.1136/bmjopen-2017-017046

5. Miesbach W, Makris M (2020) COVID-19: coagulopathy, risk of thrombosis, and the rationale for anticoagulation. Clin Appl Thromb Hemost 26:1076029620938149

6. Tang N, Bai H, Chen X et al (2020) Anticoagulant treatment is associated with decreased mortality in severe coronavirus disease 2019 patients with coagulopathy. J Thromb Haemost 18:1094-1099

7. Kreuziger L (2020) COVID-19 and VTE/Anticoagulation: frequently asked questions. Am Soc Hematol. https://www.hemat ology.org/covid-19/covid-19-and-vte-anticoagulation. Accessed 1 Sept 2020

8. Middeldorp S, Coppens M, van Haaps TF et al (2020) Incidence of venous thromboembolism in hospitalized patients with COVID-19. J Thromb Haemost 18:1995-2002. https://doi. org/10.1111/jth. 14888

9. Lodigiani C, Iapichino G, Carenzo L et al (2020) Venous and arterial thromboembolic complications in COVID-19 patients admitted to an academic hospital in Milan, Italy. Thromb Res 191:9-14. https://doi.org/10.1016/j.thromres.2020.04.024

10. Marietta M, Ageno W, Artoni A et al (2020) COVID-19 and haemostasis: a position paper from Italian Society on Thrombosis and Haemostasis [SISET]. Blood Transfus 18:167-169

11. Atallah B, Mallah SI, AlMahmeed W (2020) Anticoagulation in COVID-19. Eur Heart J Cardiovasc Pharmacother 6:260-261. https://doi.org/10.1093/ehjcvp/pvaa036

12. Gavioli EM, Sikorska G, Man A et al (2020) Current perspectives of anticoagulation in patients with COVID-19. J Cardiovasc Pharmacol 76:146-150. https://doi.org/10.1097/FJC.0000000000 000861

Publisher's Note Springer Nature remains neutral with regard to jurisdictional claims in published maps and institutional affiliations. 